Title: Gastrointestinal involvement in systemic sclerosis

Authors

Mahmoud Nassar, MD, PhD, MSc, MHA, MPA, CPHQ, SSBB.

Dr.Nassar@aucegypt.edu

0000-0002-5401-9562

Department of Medicine, Icahn School of Medicine at Mount Sinai / NYC Health + Hospitals/Queens, New York, USA

Victoria Ghernautan, MD

ghernauv@nychhc.org

0000-0002-2410-9479

Department of Medicine, Icahn School of Medicine at Mount Sinai / NYC Health + Hospitals/Queens, New York, USA

Nso Nso, MD, MPH

mcviali@yahoo.co.uk

0000-0002-0340-169X

Department of Medicine, Icahn School of Medicine at Mount Sinai / NYC Health + Hospitals/Queens, New York, USA

Akwe Nyabera, MD

anyabera1@gmail.com

0000-0002-2208-9531

Department of Medicine, Icahn School of Medicine at Mount Sinai / NYC Health + Hospitals/Queens, New York, USA

Luis Medina, MD

Dr.luismedi@gmail.com

0000-0001-9518-1470

Department of Medicine, Icahn School of Medicine at Mount Sinai / NYC Health + Hospitals/Queens, New York, USA

Wan Tu, MD

wendytu220@gmail.com

0000-0002-7363-1490

Department of Medicine, Icahn School of Medicine at Mount Sinai / NYC Health + Hospitals/Queens, New York, USA

Camelia Ciobanu, MD

kamelia.ciobanu@gmail.com

0000-0001-6556-653X

Internal medicine, Saint Barnabas Hospital, Bronx, New York, USA. 
Francisco Cuevas Castillo, MD

frankjcuevasc@gmail.com

0000-0001-7727-709X

Department of Medicine, Icahn School of Medicine at Mount Sinai / NYC Health + Hospitals/Queens, New York, USA

Vincent Rizzo MD, MBA, FACP, CPE

0000-0002-5530-447x

RIZZOV@nychhc.org

Department of Medicine, Icahn School of Medicine at Mount Sinai / NYC Health + Hospitals/Queens, New York, USA

Saphwat Eskaros, MD

Eskaros@yahoo.com

0000-0002-5119-6598

Division of Gastroenterology, Icahn School of Medicine at Mount Sinai / NYC Health + Hospitals/Queens, New York, USA

\section{Corresponding author}

Mahmoud Nassar, MD, PhD, MSc, MHA, MPA, CPHQ, SSBB.

Department of Medicine, Icahn School of Medicine at Mount Sinai / NYC Health + Hospitals/Queens, New York, USA

Email: Dr.Nassar@aucegypt.edu

Address: 82-68 164th Street Jamaica, New York 11432. 


\title{
Gastrointestinal involvement in systemic sclerosis
}

\begin{abstract}
Introduction:

The gastrointestinal tract (GI) is the second most affected organ system in systemic sclerosis or systemic/localized scleroderma (SSc) and is an important topic for research. Approximately $90 \%$ of patients with scleroderma exhibit pathology of the GI tract. The systemic scleroderma has the potential to impact any part of the gastrointestinal tract, between the oral cavity and anorectum. The pathological complications of scleroderma adversely impact the health-related quality of life of the affected patients and increase the treatment burden of patients and medical professionals.
\end{abstract}

Study Aim: We summarized the epidemiology, commonly reported clinical manifestations, complications, and available therapies for treating the GI pathology in systemic scleroderma patients.

Methodology: We performed a literature review using the keywords "systemic sclerosis," "scleroderma," "GI manifestations in scleroderma," and "GI complications of scleroderma" across databases, including Google Scholar, Medline, Embase, and PubMed. We also analyzed a range of case reports concerning scleroderma manifestations and treatment modalities.

Results: Our research revealed the annual incidence of SSc attributing to19.3 cases per million adults in the United States. We found the highest incidence of systemic scleroderma in patients within the age range of 44-55 years. Our results affirmed 5:1 incidence of systemic scleroderma that confirmed the higher impact of this disease condition in females than male populations. We found that the gastrointestinal manifestations of systemic scleroderma predominantly elevate the morbidity and mortality incidence among the affected patients. Esophageal and intestinal manifestations impact $90 \%$ and $40-70 \%$ of patients with systemic scleroderma. The small bowel hypomotility and small intestinal bacterial overgrowth (SIBO) in systemic scleroderma cases trigger the episodes of malabsorption and malnutrition that eventually add to $50 \%$ of the mortality rate. Systemic sclerosis is associated with the high incidence of fecal incontinence that triggers depression and its deleterious mental health manifestations in many clinical scenarios. 
Conclusion: The gastrointestinal complications in systemic sclerosis potentially deteriorate the daily living activities of the affected patients. The systematic management of the gastrointestinal complications of systemic scleroderma warrants multidisciplinary approaches. Prospective studies should focus on developing targeted therapies for improving the recovery patterns and prognostic outcomes in systemic scleroderma cases.

Keywords: Gastrointestinal; systemic sclerosis; scleroderma

Funding: The study was not supported by any funding sources Conflicts of interest: The authors report no relationships that could be construed as a conflict of interest. 


\section{Gastrointestinal manifestations in scleroderma}

\section{Incidence and prevalence of GI manifestations in scleroderma:}

Systemic scleroderma (SSc) is characterized by a generalized disorder of small arteries, microvessels, and connective tissues, with an annual incidence of 19.3 cases (per million adults) in the United States[1,2]. The highest incidence of systemic scleroderma occurs among individuals within the age range of 44-55 years. The female populations develop systemic scleroderma with 3-8 times higher frequency than the male populations[2]. The systemic scleroderma is highly prevalent among African Americans and women with a female-to-male ratio of 5:1[3]. Systemic Sclerosis/Scleroderma (SSc) is a rare disease condition with a prevalence of 443-660 cases per million and an incidence of 19-20 cases per million per year [3]. This progressive autoimmune condition triggers organ fibrosis, micro-vasculopathy, and gastrointestinal complications secondary to skin involvement in more than $90 \%$ of scenarios[4-6].

The patients with systemic scleroderma often report inflammation, skin fibrosis, vascular abnormalities, organ deterioration, and increased concentration of autoantibodies[7]. The systemic scleroderma also impacts the physiological functioning of the heart, lungs, kidneys, and digestive tract. The cohort study by Jaeger et al. (2016) affirmed the simultaneous onset of $50 \%$ of incidental organ manifestations in systemic scleroderma cases within two years after the onset of Raynaud's phenomenon[8]. The gastrointestinal complications in diffuse or limited scleroderma adversely impact the functioning of visceral organs[1,9]. These manifestations emanate under the impact of gastrointestinal tract fibrosis, vascular damage, and inflammatory processes[1]. The case-control study by Ciaula et al. (2008) revealed the impact of dyspepsia on diffuse gastrointestinal dysmotility and gastric antrum distension restriction in systemic scleroderma scenarios[2]. The EULAR Scleroderma Trial and Research (EUSTAR) cohort revealed 3\% of deaths from systemic scleroderma that resulted from GI complications among elderly patients and patients with diffuse skin involvement $[8,10]$. The affected patients developed a high frequency of GI symptoms at an early stage of systemic scleroderma. 
The frequency, intensity, and severity of systemic scleroderma manifestations vary with the pathology in the anorectum, esophagus, and other gastrointestinal locations[7, 9]. Increased deposition of collagen and other extracellular matrix components leads to fibrotic changes in the upper and lower GI tract, resulting in dysmotility, malabsorption, and dilation of the intestine[11]. The scleroderma symptoms commonly include meteorism, dysmotility of the esophagus, heartburn, dysphagia, nausea, vomiting, diarrhea, and constipation[7, 8, 11]. Schmeiser et al. (2012) found that amongst 90 patients with SSc, approximately $98.9 \%$ suffered from GI symptoms regardless of the stage of the disease[11]. The commonly reported symptoms of systemic scleroderma included meteorism (87.8\%) and fecal incontinence (23.5\%). Using a large-scale nationwide database, Lin et al. (2019) revealed that SSc patients exhibited a high risk of GI bleeding across gastrointestinal locations affected with peptic and non-peptic ulcers. Their findings also revealed the GI bleeding predisposition of the systematic scleroderma male patients with diabetes mellitus, hypertension, and dependence on steroids, antiplatelets, non-steroidal antiinflammatory drugs[10]. The systemic scleroderma further increases the risk for fatal manifestations, including Ogilvie syndrome or intestinal pseudo-obstruction[11]. GI symptoms of $5.3-55.6 \%$ of systemic scleroderma patients reportedly increase their risk of malnutrition[12].

The heterogeneity of symptoms in systemic scleroderma cases often masks the risk assessment interventions. The diagnostic challenges in SSC scenarios eventually barricade assessing the patient's predisposition for severe and progressive gastrointestinal disease. The heterogeneity of manifestations also restricts their correlation with immune-mediated disease activity versus the reported clinical deterioration. The diagnostic difficulties eventually challenge the medical decisions based on the administration of immunosuppressants, GI medications, and promotility agents in systemic scleroderma scenarios[9]. Additionally, increased symptom burden secondary to GI dysautonomia has been associated with higher levels of emotional distress. Studies have shown that when asked to complete the PHQ-9 Patient Health Questionnaire, depression was 25\% higher in SSc patients than in the age-adjusted healthy general population. When addressing patient needs, physical and emotional aspects must be considered to optimize the patient care processes[13]. 


\section{Morbidity and Mortality of Gastrointestinal Involvement in Systemic Sclerosis/Scleroderma:}

The standard mortality ratio of systemic scleroderma is 3-4 per million per year[14]. The cumulative death rates of $13.5 \%, 25.1 \%, 37.5 \%$, and $58.2 \%$ were reportedly recorded after the first, five, ten, and twenty years of SSc diagnosis[15]. Severe GI complications exist in 8\% of SSc patients [16]. GI tract complications prove to be the fourth common cause of mortality in $7.6 \%$ of systemic scleroderma scenarios. Other significant causes of systemic scleroderma attribute to potential complications in the lung (47.8\%), heart (25.6\%), and kidney (18.5\%) [15]. In SSc, Approximately $8.8 \%$ and $9.7 \%$ of all-cause mortality in systemic scleroderma attribute to gastrointestinal manifestations within 5 and 10 years of diagnostic affirmation[17].

The high prevalence of GI symptoms in scleroderma scenarios substantially increases the comorbidity and mortality of the affected patients. A recent study by Thoua et al. (2010) revealed 97\% of systemic scleroderma patients with upper (94\%) and lower (79\%) GI symptoms[4]. 50$90 \%$ of SSc patients exhibit esophageal dysfunction in various clinical scenarios[18]. However, scleroderma exhibits the potential to impact any part of the gastrointestinal tract[3].

The significant morbidity from commonly reported GI manifestations and/or complications are summarized in table no. 1 . The limited data based on systemic scleroderma scenarios attribute to the disease's rarity, designs of the studies, and literature review restrictions.

Steen et al. (2000) revealed severe GI tract involvement in $4 \%$ and $8 \%$ of systemic scleroderma patients at three and nine years after diagnostic affirmation[17]. The malabsorption in systemic scleroderma scenarios leads to $50 \%$ mortality after 8.5 years of diagnostic confirmation[19]. It also leads to small bowel pseudo-obstruction that leads to in-hospital mortality and mortality in 7.3$16 \%$ and $20-40 \%$ of the affected patients[16].

\section{Brief pathophysiological concepts in systemic sclerosis}

Systemic sclerosis (SSc) is a complex autoimmune disease that targets connective tissues in the skin and various internal organs in the human body. The exact etiology of SSc is unknown; however, medical literature unravels the risk factors involved in triggering the pathophysiologic processes in many clinical scenarios. The predisposing factors for SSc include long-standing 
primary Raynaud's phenomenon, stress, silicone breast implants, viruses (CMV, EBV, Parvovirus 19), and bacteria (Borrelia burgdorferi, H. Pylori) [34-36]. Together with endogenous triggers, these factors can promote specific epigenetic mechanisms in genetically predisposed individuals. The systemic sclerosis manifestations progress with microvascular damage triggered by inflammatory responses, activation of innate and adaptive immune systems, generation of autoimmune autoantibodies, and fibroblast activation leading to extensive tissue fibrosis [34, 37 , $38]$.

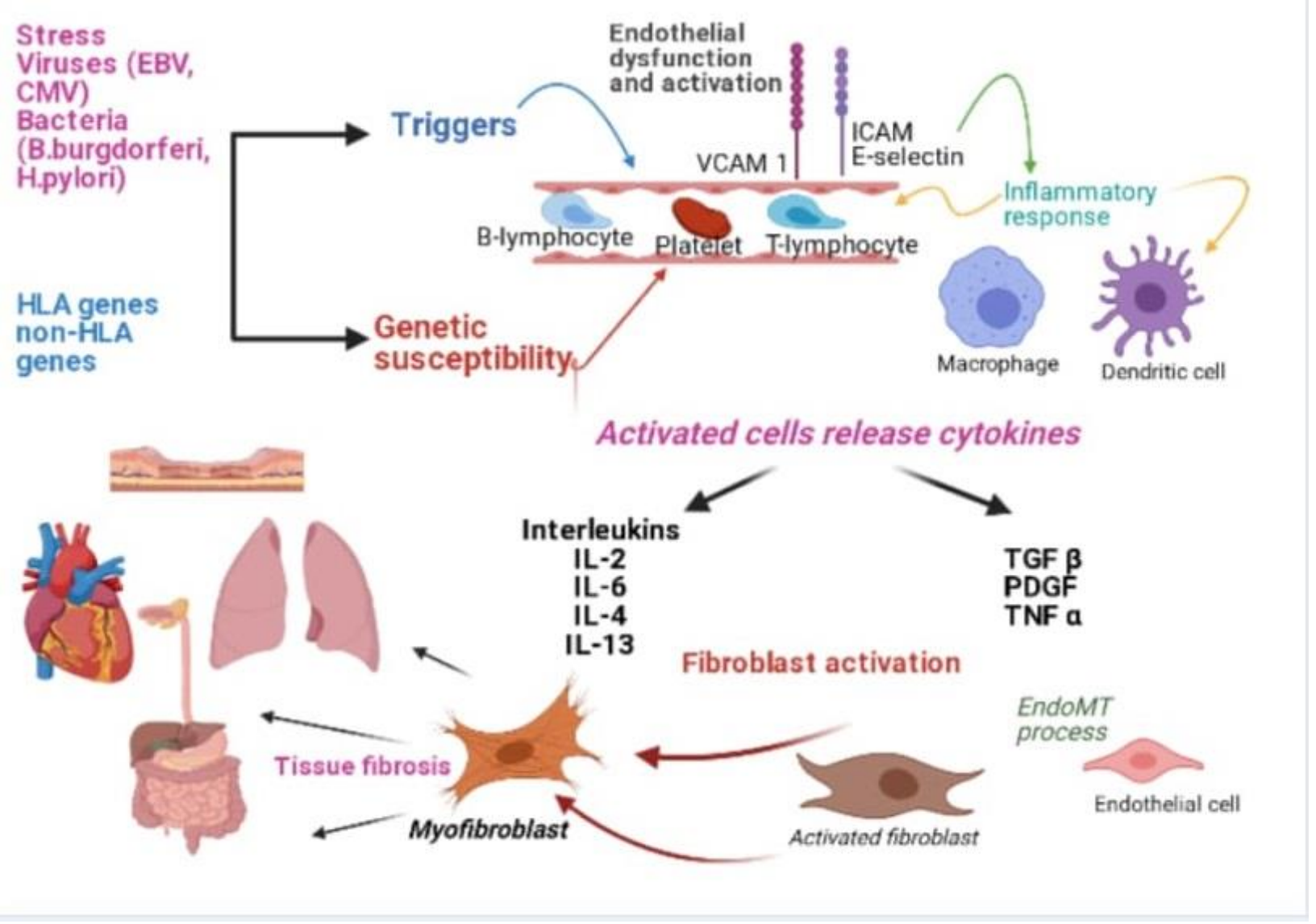

Figure 1: Microvascular injury, inflammatory and autoimmune responses

The long-standing Raynaud's phenomenon (RP) actively progresses in more than $95 \%$ of systemic scleroderma patients. The long-standing RP eventually progresses to secondary RP, followed by the clinical manifestations in other organs in systemic scleroderma scenarios. The systemic scleroderma progressively develops under the impact of endothelial dysfunction and a cascade of 
events resulting in an imbalance of vasoconstrictor and vasodilator factors, including ET-1, NO, and prostacyclin. Vasoconstriction in systemic scleroderma leads to the thickening of the vessel wall and luminal narrowing that eventually reduced the number of capillaries, thereby triggering tissue hypoxia $[34,39,40]$. The vascular damage predominantly impacts the small and mediumsized vessels of the skin, lungs, heart, kidney, and GI tract of the systemic scleroderma patients[35]. The deterioration of the small/medium-sized blood vessels eventually triggers the proangiogenic factors (VGEF-A) for new angiogenesis. However, the imbalance between proangiogenic and antiangiogenic mediators restricts the angiogenetic response processes in systemic scleroderma patients[34, 35]. However, the patients with systemic sclerosis report a marked increase in their angiogenetic mediators or VEGF $165 \mathrm{~b}$ serum levels. However, the selective binding of VEGF $165 \mathrm{~b}$ with VEGF-2 receptor across the endothelium impairs angiogenesis in systemic scleroderma patients[34].

The repeated alterations of the microvascular tone result in impairment of the endothelial barrier and activation of the endothelial cells that eventually increases the expression of adhesion molecules like vascular cell adhesion protein 1 (VCAM1), intercellular adhesion molecule (ICAM), E-selectin further increases, which promotes inflammatory and immune cells recruitment, their migration into interstitial space, and tissue infiltration [34, 41]. These processes also impact the functionality of macrophages, monocytes, platelets, dendritic cells, B and $\mathrm{T}$ lymphocytes, fibroblasts, myofibroblasts. The activated cells produce cytokines (including IL-2, Il-4, IL-6, IL-13, transforming growth factor B TGF $\beta$, platelet-derived growth factor PDGF, tumor necrosis factor $\mathrm{TNF} \alpha$ ) that stimulate fibroblast proliferation and fibrosis [40, 42, 43]. The type 2 $\mathrm{T}$ helper cells exist in a higher proportion than type $1 \mathrm{~T}$ helper in SSc patients, while the profibrogenic IL-4 and IL 13 are secreted by type 2 T helper cells [34, 36, 44]. B cell involvement results in autoantibodies production directed against nuclear, cytoplasmic, and extracellular autoantigens. The autoantibodies that exhibit a high specificity to diagnose systemic scleroderma include anti-topoisomerase AAbs, anti-RNA polymerase I/III AAbs, anticentromere AAbs, and anti-Th/To ribonucleoprotein AAbs. These antibodies indirectly correlate with scleroderma pathogenesis in many clinical scenarios [34, 42, 44].

\section{Tissue fibrosis-The hallmark of pathogenesis in Systemic Sclerosis}


The fibrogenesis process in systemic sclerosis relies on the overactivation of fibroblasts by TGF $\beta$, ET-1, vasculopathy, and loss of their homeostatic state leading to their trans-differentiation in peripheral tissue into metabolically active myofibroblasts [39, 44, 45]. The endothelial cells express mesenchymal cell products ( $\alpha$-smooth muscle actin, collagen type I) during the endothelial-to-mesenchymal transition process (Endo-MT) in systemic sclerosis and eventually acquire mesenchymal/myofibroblast characteristics. Local fibroblasts and circulating fibrocytes (fibroblast-like cells) are a potential source of myofibroblasts. The activation of fibroblasts and myofibroblasts contributes to excessive collagen deposition, promoting tissue fibrosis in the skin, lungs, heart, and GI tract of the systemic scleroderma patients [34, 44].

\section{Management of GI manifestations in systemic scleroderma: Investigations and treatment}

\section{Oral cavity}

Approximately $68 \%$ of patients with systemic scleroderma (SSc) experience Sicca syndrome based on morbidities including, polymyositis, scleroderma, lupus, rheumatoid arthritis, dry mouth, and dry eyes [46]. The labial minor salivary gland biopsy is the recommended prognostic tool to evaluate the risk of lymphoma in patients with systemic scleroderma. This biopsy helps determine the presence of germinal center lesions (via light microscopy) that impact 5\% of SSc patients with a high predisposition for lymphoma [47].

The systemic scleroderma patients with Sicca syndrome require an intraoral assessment to evaluate their risk for mandibular bone resorption, caries, and periodontitis [48]. These patients further require health education to increase their dental care and salivary secretions in the context of reducing their mouth dryness. However, the treatment strategies include rehabilitation via orofacial exercises and administration of cevimeline, pilocarpine, muscarinic agonists, and artificial saliva [49-51].

\section{Esophagus}

Esophageal involvement occurs almost in all patients with systemic sclerosis[52]. The investigation and management target the dysmotility disorder and its manifestations, including dysphagia, gastrointestinal reflux disease, heartburn, and regurgitation. Manometry further helps evaluate dysphagia and its clinical complications. The typical findings include absent peristalsis, 
ineffective peristalsis, and hypotensive lower esophageal sphincter pressure[53, 54]. Recently, high-resolution manometry is a preferred diagnostic approach due to its potential to detect esophageal dysmotility in asymptomatic patients[55] despite its controversial role in clinical practice[56]. The esophageal $\mathrm{pH}$ monitoring is mainly performed on patients with resistant reflux symptoms or lung transplant candidates[57].

Esophagogastroduodenoscopy (EGD) effectively diagnoses reflux-related esophagitis, esophageal dysmotility, H pylori infection, esophageal strictures, Barrett esophagus, and adenocarcinoma. A retrospective study performed on asymptomatic patients confirmed reflux-esophagitis in $77 \%$ of cases on EGD. The study findings also revealed distal esophageal dysmotility in $85 \%$ of patients, gastritis in $92 \%$ of patients, and Helicobacter pylori infection in $38 \%$ of patients. These findings emphasize the significance of early detection and management in improving the prognostic outcomes in systemic scleroderma scenarios [58].

The systematic management of GERD (gastroesophageal reflux disease) and esophageal motility disorder warrants multifaceted approaches relying on lifestyle management [115]. The preliminary measures include head elevation at night, exclusion of triggering foods/substance abuse, and consumption of small/frequent meals during the day. The proton pump inhibitors (PPIs) are the standalone medications for the clinical management of GERD. PPIs further improve clinical symptoms and prevent esophageal complications in patients with systemic sclerosis[59]. In patients with modest clinical response to daily or twice daily PPIs, an $\mathrm{H} 2$ receptor inhibitor taken at bedtime, The reduction in nighttime reflux symptoms and GERD-associated sleep disturbances in SSc scenarios warrants the administration of PPIs (twice daily) and H2 receptor inhibitor (at bedtime) $[60,61]$. The inclusion of prokinetic drugs including cisapride, domperidone, and metoclopramide in the PPI regimen may further enhance the therapeutic outcomes in systemic sclerosis patients with esophageal dysmotility or partial response to PPI management. The therapeutic approaches based on PPIs and prokinetic medications effectively reduce GERD manifestations while improving gastric emptying, esophageal sphincter pressure, and intestinal peristalsis[62-64]. The endoscopic dilatation and continuous administration of include the preferred therapies for the systematic management and prevention of esophageal strictures in systemic sclerosis.EGD surveillance with biopsy is recommended in Barrett's esophagus, and 
treatment includes endoscopic ablation or resection of dysplastic epithelium using photochemical, thermal, or radio ablation energy[65].

\section{Stomach}

Gastroparesis in systemic sclerosis leads to early satiety, nausea/vomiting, abdominal pain, bloating, and distention. The assessment of delayed gastric emptying via scintigraphy in SSc scenarios helps diagnose the onset of gastroparesis. EGD further assists in ruling out gastric outlet obstruction and Helicobacter pylori infection or gastritis in SSc patients with gastroparesis. The first-line therapy for gastroparesis relies on dietary modifications that necessitate the frequent intake of low fat/fiber-based diet and vitamin supplementations. The potential of prokinetics in the clinical management of gastroparesis appears questionable in the absence of their safety and efficacy profiles. However, the medical literature supports the use of metoclopramide (liquid formulation for 12 weeks) for treating gastroparesis[66, 67]. Clinical studies also report the adverse effects of metoclopramide following its prolonged use among patients with systemic sclerosis. Other off-label medications with the potential to manage gastroparesis in systemic sclerosis include domperidone, erythromycin, cisapride, and ghrelin agonists[68, 69]. The administration of antiemetics, however, provides symptomatic relief while preventing nausea and vomiting in SSc patients. More invasive procedures such as jejunostomy tube or gastrostomy tube for feeding and stomach decompression may be considered in patients who experience refractory symptoms after receiving pharmacotherapy. A laparoscopic or endoscopic pyloroplasty is rarely performed for managing gastroparesis in SSc scenarios[70]. The medical literature provides weak evidence regarding the role of gastric electrical stimulation in controlling abnormal rhythms, stimulate gastric emptying, and providing symptomatic relief in systemic sclerosis patients with gastroparesis[69, 71].

In patients with symptomatic anemia or suspected occult gastrointestinal bleeding, EGD is the gold standard of care due to its potential to diagnose vascular lesions, small telangiectasias, gastric antral vascular ectasia (GAVE), or so-called "watermelon stomach." The endoscopic intervention helps diagnose gastric antral vascular ectasia by tracking the longitudinal flat rows from the pylorus to the antrum[72]. The recommended treatment options for GAVE include endoscopic coagulation with laser therapy, argon plasma coagulation, and radiofrequency intervention in 
refractory cases [73]. However, the treatment of symptomatic anemia in systemic scleroderma cases relies on intermittent blood transfusions and iron supplementation.

\section{Small intestine}

The cross-sectional imaging of the small intestine is the method of choice to evaluate pseudoobstruction in the abdomen. The cross-sectional imaging assists in evaluating dilatation in the small intestine in the absence of mechanical obstruction. However, the treatment modalities include prokinetics like cisapride[74], metoclopramide, and domperidone in conjunction with antibiotics[75]. Medical literature also reveals the efficacy of octreotide in improving abdominal symptoms and stimulating intestinal motility in patients with systemic scleroderma[76]. The gold standard for diagnosing SIBO (small intestinal bacterial growth) relies on the microbial culture of jejunal aspirates. The diagnostic affirmation depends on the finding of greater than $10^{3}$ colony factor unit[77]. The hydrogen (glucose or lactulose) and methane breath tests are largely utilized in clinical settings due to their noninvasive nature and easy availability. The positivity of the breath test relies on the hydrogen concentration of $\geq 20$ parts per million (ppm) during the 90 minutes glucose or lactulose challenge and an increase in methane levels to $\geq 10 \mathrm{ppm}[77]$. The $60 \%$ sensitivity and $80 \%$ specificity of the breath test make it a favorable diagnostic option for SIBO assessment [78, 79]. The majority of the systemic scleroderma patients exhibit nutritional deficiency, malnutrition, and weight loss requiring evidence-based management via nutritional support and hydration.

The symptomatic treatment of small intestinal bacterial growth relies on systematic selection and administration of antibiotics [80]. The absence of a standard therapeutic regimen for SIBO warrants its systematic management based on culture findings, the severity of symptoms, and clinical response. Rifaximin is a frequently reported antibiotic in meta-analysis studies demonstrating clinical effectiveness against SIBO in systemic sclerosis settings[80-82]. Other antibiotics with therapeutic efficacy for SIBO include ciprofloxacin, norfloxacin, amoxicillin, tetracyclines (doxycycline), metronidazole, and trimethoprim-sulfamethoxazole[83-85]. The therapeutic management of SIBO warrants the treatment duration of 10-14 days based on the severity of reported symptoms. The meta-analysis by Zhong et al. (2017) affirms the therapeutic 
efficacy of probiotics in terms of alleviating abdominal pain and other intestinal manifestations during SIBO in systemic sclerosis[86].

\section{Colon and anorectal involvement}

Approximately $20 \%-50 \%$ of patients with systemic sclerosis report intestinal manifestations based on diarrhea and constipation. Constipation in systemic sclerosis progresses due to slow intestinal transit based on muscle atrophy and neuropathy. The diagnostic assessment relies on digital rectal exam and CT or abdominal radiography in patients with colonic pseudo-obstruction, dilatation or impaction, volvulus, megacolon, or perforation. Colonoscopy effectively evaluates the etiology of cancers and lower GI bleeding (telangiectasia) in patients with systemic sclerosis.

The empirical treatment measure for systemic sclerosis includes administering stimulant laxatives and stool softeners for constipation management. The new secretory agents including, lubiprostone, linaclotide, and Plecanatide[87-89]. Effectively treat chronic constipation; however, their safety and efficacy appear questionable in systemic sclerosis scenarios. Prokinetic agents, including prucalopride, also exhibit therapeutic efficacy for constipation in systemic sclerosis[90].

Diarrhea in patients with systemic sclerosis requires multifactorial approaches for its clinical management. The Clostridium difficile is a preferred diagnostic modality to rule out infectious manifestations in systemic sclerosis. The differential diagnoses including bile acid malabsorption, fructose intolerance, SIBO, and amyloidosis correlate with persistent colonic inflammation in systemic sclerosis. The treatment guided by etiology effectively challenges the pathology of systemic sclerosis and improves its prognostic outcomes. The first-line therapy to manage the colonic manifestations in systemic sclerosis includes dietary modification or targeted dietary therapy based on FODMAP administration (Fermentable Oligosaccharides, Disaccharides, Monosaccharides, and Polyols)[91, 92]. The selective and cautious use of loperamide also assists in managing diarrhea in patients with systemic sclerosis.

$20-40 \%$ of systemic sclerosis patients develop fecal incontinence seen in approximate 20-40\%[93, 94] that manifests involuntary soiling via recto-anal inhibitory reflex and a decrease in internal anal resting tone[95]. The high severity of fecal incontinence in systemic sclerosis patients substantially deteriorates their health-related quality of life and quality-adjusted life years[96]. 
Diagnostic modalities performed in individuals with suspected fecal incontinence include anorectal manometry, magnetic resonance (MR) defecography, endoanal ultrasound, and balloon expulsion test [97]. The treatment options include dietary modifications, anti-diarrheal medications, and antibiotic treatment in case of concomitant SIBO. Anorectal biofeedback training displays encouraging results in improving symptoms and health-related quality of life[98]. In more severe circumstances, sacral nerve stimulation is the preferred treatment option based on its safety and efficacy in systemic scleroderma cases[99].

\section{New treatments and recommendations in GI manifestations in scleroderma}

Systemic sclerosis predominantly impacts the GI tract of the affected patients. The gastrointestinal manifestations reportedly occur in $90 \%$ of patients with systemic sclerosis. Systemic sclerosis substantially deteriorates esophageal functioning in most cases; however, it also triggers gastroesophageal reflux (GER), esophageal dysmotility, strictures, pancreas, and hepatopancreatic manifestations [27].

The recommended treatment modalities for managing gastrointestinal manifestations in scleroderma cases must be supported by dietary modifications for improving the prognostic outcomes. The dietary modifications (for improving the digestive processes) rely on the administration of probiotics, low-fermentable oligosaccharides, disaccharides, monosaccharides, and polyols (low-FODMAP). The exclusion of smoking habits is further recommended to reduce the risk of clinical adversities [100]. In 2016, the EULAR released the updated recommendations for treating systemic sclerosis [101]. The experts recommended the administration of Proton pump inhibitors (PPIs) in symptomatic patients with GER to prevent their gastrointestinal complications. The coadministration of $\mathrm{H} 2$ blockers and sucralfate with PPIs in many scenarios assists the management of gastrointestinal complications in systemic sclerosis. The individualization of combination therapies, however, depends on the intensity and severity of the reported gastrointestinal complications. The use of surgical procedures like Nissen fundoplication or the Roux-en-Y can also be considered on a case-to-case basis. The use of PPIs in asymptomatic patients is controversial, given the side effects of long-term PPI use [101].

Systemic sclerosis triggers motility dysfunction in the entire GI tract that warrants the administration of PPIs and prokinetic drugs including, metoclopramide, erythromycin, 
domperidone, and cisapride. The prokinetic medicines and PPIs effectively increase the lower esophageal sphincter (LES) pressure and peristaltic amplitude in the distal esophageal body. The open-label study by Frech (2018) revealed the therapeutic efficacy of oral 5-hydroxytryptamine 1A (5-HT1A) receptor agonist (Buspirone) in terms of increasing the LES resting and reducing the severity of heartburn and regurgitation over four weeks [97].

The gastric dysmotility treatment relies on prokinetic drugs administered for esophageal dysmotility. The novel treatment option includes Gastric Per-Oral Endoscopic Pyloromyotomy (G-POEM) that may increase the therapeutic outcomes in systemic scleroderma patients with failed medical therapy. The treatment goal for managing gastric antral vascular ectasia (GAVE) includes reducing bleeding and obliteration of ectasia via radiofrequency ablation, laser therapy, or endoscopic coagulation with argon plasma coagulation intervention[102].

The peristaltic dysfunction in the small bowel the small intestinal bacterial overgrowth (SIBO) eventually leads to malabsorption and malnutrition in patients with systemic sclerosis. The treatment modality relies on administering antibiotics between 14-21 days based on the severity of diarrhea and other intestinal complications. Medical literature recommends daily therapy with alternating antibiotics every two weeks to minimize the risk of relapsing disease. The clinical studies also recommend the use of probiotics for treating the episodes of abdominal distension and bloating in systemic scleroderma[27]

The management of constipation in systemic sclerosis relies on the administration of stool softeners, a high-fiber diet, and probiotics. Prucalopride, a 5HT4 receptor agonist, is a possible therapeutic option based on its potential to improve gastric emptying and proximal colonic motility in systemic scleroderma. Medical literature also supports the therapeutic efficacy of sacral nerve stimulation to improve fecal incontinence and anorectal function in systemic sclerosis. The bulking agents, anti-diarrheal medicines, bile acid-binding resins, and sphincter motor training or biofeedback interventions effectively reduce stool frequency and improve stool consistency in patients with systemic sclerosis [27].

The liver involvement in SSc is rare and usually related to autoimmune hepatitis and primary biliary cholangitis (PBC). Its management depends on the use of prednisone and other medications that antagonize the risk of therapy-induced hepatotoxicity in systemic scleroderma. The 
development of pancreatic disease in systemic sclerosis follows the exocrine pancreatic insufficiency requiring enzyme supplementation [27, 97].

Cyclophosphamide, Methotrexate, and Mycophenolate Mofetil include the immunosuppressants that effectively treat the vascular, pulmonary, and skin manifestations of systemic scleroderma. The biological medications, anti-fibrotic, and small molecules like Tyrosine kinase inhibitors, rapamycin, and pamidronate also exhibit the therapeutic potential to control the GI manifestations in systemic sclerosis. However, the medical literature does not validate any standard therapy for the medical management of advanced fibrosis in systemic scleroderma. The clinical studies provide limited evidence in favor of the therapeutic efficacy of Intravenous Immunoglobulin (IVIG) for managing the gastrointestinal manifestations in systemic sclerosis. The prospective large clinical trials addressing the effect of these novel therapies on the GI tract are needed to optimize the treatment interventions for systemic scleroderma[103].

\section{Complications of GI manifestations in scleroderma}

The gastrointestinal complications in systemic scleroderma potentially impact the quality of life and elevate the risk of mortality [104]. Their therapeutic management proves highly challenging in a variety of clinical scenarios. More than $90 \%$ of patients with systemic scleroderma report GI tract pathology and its clinical manifestations[104, 105]. The GI complications of scleroderma potentially trigger serious clinical complications that deteriorate the structure and function of the intestine, gastric region, esophagus, oropharynx, and visceral organs. The high prevalence of esophageal and bowel manifestations in systemic scleroderma substantially deteriorates the quality-assisted life years and survival rate of the affected patients[91, 105].

The oropharyngeal complications in systemic scleroderma emanate from oral and perioral tissue fibrosis, chronic inflammation, histopathological and anatomical changes (due to atrophy), oral cavity disfigurement, and malalignment of osseous structures leading to microstomia and teeth malocclusion. The systemic sclerosis patients may eventually experience impaired mastication and deglutition, food leakage, regurgitation, voice hoarseness, and aspiration [97, 106]. Approximately one-fifth of systemic sclerosis patients experience Secondary Sjogren Syndrome, leading to loss of teeth due to dental caries and periodontal diseases [104, 107]. 
Approximately 50 to $90 \%$ of patients with scleroderma experience esophageal manifestations[22, 97]. The early identification of esophageal complications in scleroderma cases is necessary to reduce the risk of fatal manifestations. The complications, including organ dysfunction and fibrosis in scleroderma cases, emanate from microvascular changes and inflammatory manifestations in connective tissues, muscles, and nerves. The esophageal complications in systemic scleroderma potentially reduce esophageal peristalsis and decreasing the lower sphincter pressure. The esophageal manifestations in systemic scleroderma trigger the development of GERD, reflux esophagitis, esophageal stricture Barrett's esophagus, and adenocarcinoma[29, 105, 108]. The acid reflux further triggers erosive/hemorrhagic esophagitis, leading to esophageal ulcers in patients with systemic scleroderma. The lack of treatment of esophageal complications in systemic sclerosis patients increases the risk of achalasia-like syndrome, Barrett's esophagus, and adenocarcinomas [109]. Approximately $12.7 \%$ of patients with systemic sclerosis with 2-year endoscopy status develop Barrett's esophagus and its serious complications [24]. The estimated incidence of Barrett's esophagus in systemic sclerosis attributes 6.8 to $12.7 \%$ compared to less than $1 \%$ for the general population. The systemic scleroderma patients with severe symptoms of Barrett's esophagus experience a high predisposition for adenocarcinoma, esophageal and oropharyngeal cancers than the general population [109].

Approximately $38-50 \%$ of patients with systemic scleroderma experience an elevated predisposition for gastric dysmotility that eventually leads to gastroparesis. Severe gastroparesis with persistent nausea and vomiting in systemic scleroderma cases triggers dehydration and electrolyte abnormalities [105]. Gastric Antral Vascular Ectasia (GAVE), also known as "watermelon stomach," is a rare clinical condition associated with various pathologies, including systemic scleroderma. GAVE in systemic sclerosis potentially triggers chronic gastrointestinal bleeding and iron deficiency anemia $[105,110]$.

The intestinal pathology in scleroderma adversely impacts the health-related quality of life and requires multidisciplinary management. The intestinal manifestations in systemic scleroderma lead to small bowel and colonic complications. The small bowel dysmotility in systemic sclerosis patients increases their risk of small intestinal bacterial overgrowth. Their intestinal hypomotility further induces lumen dilatation and pseudo-obstruction of the intestine. The rare complication of systemic sclerosis includes bowel wall necrosis and perforation[105]. Malnutrition in systemic 
sclerosis progresses via disrupted digestion and malabsorption. Malnutrition and weight loss in systemic sclerosis also develop under the impact of environmental and genetic factors. The decrease in oral food intake in systemic sclerosis often triggers nausea, vomiting, dysphagia, and perioral changes. In many scenarios, the patients with systemic sclerosis also develop contractures of fingers that eventually impair their meal preparation and eating activities. The appetite reduction in systemic sclerosis patients also correlates with their depressive manifestations[109, 111]. The malnutrition in systemic sclerosis adds to the disease severity, poor prognosis, and increased mortality rate $[112,113]$.

The colonic and anorectal complications lead to a variety of presentations in systemic sclerosis. Constipation in systemic sclerosis is a primary manifestation that indicates colonic involvement and leads to severe complications, including megacolon, ulceration, and volvulus. The fecal incontinence in systemic sclerosis is a consequence of fibrosis and atrophy of the internal anal sphincter that eventually decreases the resting anorectal pressures. Anal sensory neuropathy plays a pivotal role in fecal incontinence among systemic sclerosis patients [91]. The fecal incontinence predominantly elevates mood swings and depressive episodes in systemic sclerosis scenarios [105]. Pneumatosis cystoides intestinalis is a rare complication that develops in patients with systemic sclerosis. The systemic sclerosis patients with pneumatosis cystoides intestinalis develop gas-filled cysts in the subserosa and submucosa of their small or large intestines. They also report abdominal pain or flatulence and change in bowel habits[29, 91].

Funding: The study was not supported by any funding sources.

Conflicts of interest: The authors report no relationships that could be construed as a conflict of interest. 
Table 1: Prevalence, complications, and diagnostic management of GI manifestations in systemic sclerosis patients

\begin{tabular}{|c|c|c|c|}
\hline Organ involvement & Prevalence & $\begin{array}{c}\text { Gastrointestinal } \\
\text { manifestations/complications } \\
\text { and their prevalence }\end{array}$ & Diagnosis/Management \\
\hline $\begin{array}{l}\text { Oropharyngeal } \\
\text { involvement }\end{array}$ & $10-70 \%{ }^{[1]}$ & $\begin{array}{l}\text {-Microstomia- } 43-80 \%^{[2]} \\
\text {-Xerostomia and periodontal } \\
\text { disease- } 30-73 \%{ }^{[2]} \\
\text {-Gingival inflammation/bleeding- } \\
60-73 \%{ }^{[2-4]} \\
\text {-Oropharyngeal dysphagia- } 25 \%\end{array}$ & $\begin{array}{l}\text { 1. Regular dental exams }{ }^{[3]} \text {. } \\
\text { 2. Panoramic radiographic exams to assess for } \\
\text { osseous changes }^{[1,3]} \text {. } \\
\text { 3. Good oral hygiene and artificial } \\
\text { saliva/lubricants to manage dental and } \\
\text { oral/perioral soft tissue pathology } y^{[1,3]} \text {. } \\
\text { 4. Mechanical soft foods, small bolus size, } \\
\text { mouth stretching, even bilateral } \\
\text { commissurotomy are treatment options } \\
\text { for decreased mouth opening }\end{array}$ \\
\hline $\begin{array}{l}\text { Esophageal } \\
\text { involvement }\end{array}$ & $\begin{array}{l}90 \% ; 30-50 \% \\
\text { can be } \\
\text { asymptomatic } \\
{[19,24,27,} \\
59]\end{array}$ & $\begin{array}{l}\text {-GERD } 90 \%{ }^{[5]} \\
\text {-Lower esophageal sphincter laxity } \\
37.8-55 \%{ }^{[5,6]} \\
\text {-Esophagitis } 60 \%{ }^{[7]} \\
\text {-Esophageal strictures } 41 \%^{[7]} \\
\text {-Barrett's esophagus } 12.7-13 \%{ }^{[8]}\end{array}$ & $\begin{array}{l}\text { 1. EGD is used for diagnosing esophagitis, } \\
\text { Barrett's, adenocarcinoma }{ }^{[9]} \text {. } \\
\text { 2. Esophageal manometry is used for the } \\
\text { evaluation of esophageal dysmotility }{ }^{[1,9]} \text {. } \\
\text { 2. Barium swallow can be used to detect } \\
\text { strictures and their severity }{ }^{[9]} \text {. } \\
\text { 3. pH monitoring to assess for therapeutic } \\
\text { efficacy of PPI in GERD }{ }^{[1,9]} \text {. } \\
\text { 4. PPI is the mainstem of treatment of acid } \\
\text { suppression required in scleroderma patients } \\
\text { to relieve GERD symptoms and prevent } \\
\text { complications. } \\
\text { 5. Lifestyle modification is also } \\
\text { recommended (avoidance of large and } \\
\text { late-night meals) }{ }^{[1,3]} \text {. }\end{array}$ \\
\hline
\end{tabular}




\begin{tabular}{|c|c|c|c|}
\hline Gastric involvement & $50 \%{ }^{[10]}$ & $\begin{array}{l}\text {-Gastroparesis 50\% } \\
\text {-Gastric Antral Vascular Ectasia } \\
\text { (GAVE) 5.6-22.3\% }{ }^{[3,10]} \\
\text { Gastric Bleeding Ectasis } 0.6-0.8 \% \\
\text { [11] } \\
\text {-Upper GI Bleeding 3.2\% }\end{array}$ & $\begin{array}{l}\text { 1. Gastric emptying study }{ }^{[1,3,9]} \text {. } \\
\text { 2. EGD is used to diagnose GAVE if the } \\
\text { patient has iron deficiency anemia and for } \\
\text { therapeutic purposes like laser } \\
\text { photocoagulation or endoscopic band } \\
\text { ligation }{ }^{[1,3,9]} \text {. } \\
\text { 3. Pro-kinetics (metoclopramide, } \\
\text { domperidone) are used for gastroparesis }^{\text {management }}{ }^{[3]} \text {. }\end{array}$ \\
\hline $\begin{array}{l}\text { Small bowel } \\
\text { involvement }\end{array}$ & $\begin{array}{l}40 \% ; 20 \% \\
\text { can be } \\
\text { asymptomatic } \\
{[3,13]}\end{array}$ & $\begin{array}{l}\text {-Diarrhea- 27.7-79\% }{ }^{[3,14]} \\
\text {-Small Intestinal Bacterial } \\
\text { Overgrowth } 33-50 \%^{[10]} \\
\text {-Malabsorption } 10-25 \%^{[10]} \\
\text {-Small Bowel Pseudo-obstruction- } \\
\text { 5.4\% }{ }^{[15]} \\
\text {-Pneumatosis Cystoides } \\
\text { Intestinalis Rare }{ }^{[3,16]}\end{array}$ & $\begin{array}{l}\text { 1. Scintigraphy, capsule endoscopy, MRI/CT } \\
\text { enterography may be performed to evaluate } \\
\text { small bowel involvement and extent }{ }^{[3]} \text {. } \\
\text { 2.Hydrogen-breath test to assess for SIBO }{ }^{[3,9,} \\
\text { 16]. } \\
\text { 3. C. Diff testing, stool studies may be } \\
\text { indicated in patients with diarrhea }{ }^{[3,9]} \text {. } \\
\text { 4. Abdominal DX and CT abdomen for } \\
\text { pseudo-obstruction evaluation }{ }^{[9]} \text {. } \\
\text { 5. Measurement of fat-soluble vitamins if } \\
\text { malabsorption is suspected }{ }^{[9]} \text {. } \\
\text { 6. Diet modification, probiotics, and } \\
\text { antibiotics (fluoroquinolones, metronidazole, } \\
\text { tetracycline, rifaximin) are treatment options } \\
\text { for SIBO }{ }^{[1,3]} \text {. }\end{array}$ \\
\hline Colon involvement & $20-50 \%[3,10]$ & $\begin{array}{l}\text {-Constipation } 9.2-38 \%^{[3,14]} \\
\text {-Megacolon } 1.5-3.8 \%{ }^{[14]} \\
\text {-Large intestine vascular ectasia } \\
1.3-3.1 \%{ }^{[14]} \\
\text {-Lower GI bleeding } 2.9 \%{ }^{[12]} \\
\text {-Wide-mouth diverticula } 1.3-8.6 \% \\
{[14,17]}\end{array}$ & $\begin{array}{l}\text { 1.Colonoscopy is recommended for } \\
\text { scleroderma patients with new-onset } \\
\text { constipation }^{[3,9,16]} \text {. } \\
\text { 2.Fiber supplementation, bowel training, stool } \\
\text { softeners, laxatives, prokinetics are } \\
\text { recommended to manage constipation }{ }^{[1,3,16]} \text {. }\end{array}$ \\
\hline $\begin{array}{l}\text { Anorectal } \\
\text { involvement }\end{array}$ & $\begin{array}{l}50-70 \%{ }^{[3,7,} \\
16]\end{array}$ & $\begin{array}{l}\text {-Rectal Prolapse } 20 \%{ }^{[18]} \\
\text {-Fecal Incontinence } 20-38 \%[10,14, \\
\text { 19] }\end{array}$ & $\begin{array}{l}\text { 1. Anorectal manometry, MR defecography, } \\
\text { balloon expulsion test is used to assess for } \\
\text { fecal incontinence }{ }^{[9,16]} \text {. }\end{array}$ \\
\hline
\end{tabular}




\begin{tabular}{|c|c|c|c|}
\hline & & -Fecal Impaction $18 \%{ }^{[14]}$ & $\begin{array}{l}\text { 2. Antidiarrheal agents and diet changes are } \\
\text { used to improve stool consistency [29] } \\
\text { 3. Sacral nerve stimulation was shown to be } \\
\text { beneficial in the management of fecal } \\
\text { incontinence }{ }^{[3,16]} \text {. } \\
\text { 4. Surgical intervention may be indicated } \\
\text { for rectal prolapse }{ }^{[1,16]} \text {. }\end{array}$ \\
\hline Liver involvement & $1.1-1.5 \%$ & $\begin{array}{l}\text { Primary biliary cirrhosis (PBC) } 2- \\
18 \%{ }^{[20]} \\
\text { Autoimmune hepatitis-rare } \\
{[3,9]}\end{array}$ & $\begin{array}{l}\text { 1. Checking liver enzymes, bilirubin, and } \\
\text { antimitochondrial antibodies }{ }^{[1,3]} \text {. } \\
\text { 2. If PBC is suspected but AMA negative, } \\
\text { anti-gp } 210 \text { and anti-sp100 are highly } \\
\text { specific }^{[3]} \text {. } \\
\text { 3. Liver ultrasound if the tests are } \\
\text { abnormal }^{[1]} \text {. } \\
\text { 4. Avoidance of hepatotoxic medications. } \\
\text { 5. Hepatic dosing of medications } \\
\text { metabolized by the liver }^{[9]} \\
\text { 6. Ursodeoxycholic acid for PBC }{ }^{[1,3,9]} \text {. }\end{array}$ \\
\hline
\end{tabular}




\section{References:}

1 Savarino E, Furnari M, de Bortoli N, Martinucci I, Bodini G, Ghio M, Savarino V. Gastrointestinal involvement in systemic sclerosis. Presse Med. 2014: e279 [PMID: 25179275

10.1016/j.Ipm.2014.03.029: 10.1016/j.Ipm.2014.03.029]

2 Yuen HK, Weng Y, Reed SG, Summerlin LM, Silver RM. Factors associated with gingival inflammation among adults with systemic sclerosis. International journal of dental hygiene. 2014: 55

3 McFarlane IM, Bhamra MS, Kreps A, Iqbal S, Al-Ani F, Saladini-Aponte C, Grant C, Singh S, Awwal K, Koci K. Gastrointestinal manifestations of systemic sclerosis. Rheumatology (Sunnyvale, Calif). 2018:

4 Scardina GA, Messina P. Systemic sclerosis: description and diagnostic role of the oral phenomena. General dentistry. 2004: 42

5 Denaxas K, Ladas SD, Karamanolis GP. Evaluation and management of esophageal manifestations in systemic sclerosis. Annals of gastroenterology. 2018: 165

6 Markus J, Pinto RMC, Matoso AGB, Ranza R. Esophageal manometry in systemic sclerosis: findings and association with clinical manifestations. Rev Assoc Med Bras (1992). 2020: 48 [PMID: 32130381

10.1590/1806-9282.66.1.48: 10.1590/1806-9282.66.1.48]

7 Ntoumazios SK, Voulgari PV, Potsis K, Koutis E, Tsifetaki N, Assimakopoulos DA. Esophageal involvement in scleroderma: gastroesophageal reflux, the common problem. Semin Arthritis Rheum. 2006: 173 [PMID: 17045629 10.1016/j.semarthrit.2006.08.002: 10.1016/j.semarthrit.2006.08.002] 8 Wipff J, Allanore Y, Soussi F, Terris B, Abitbol V, Raymond J, Chaussade S, Kahan A. Prevalence of Barrett's esophagus in systemic sclerosis. Arthritis \& Rheumatism. 2005: 2882

9 Frech TM, Mar D. Gastrointestinal and hepatic disease in systemic sclerosis. Rheumatic Disease Clinics. 2018: 15

10 Gyger G, Baron M. Systemic sclerosis: gastrointestinal disease and its management. Rheumatic Disease Clinics. 2015: 459

11 Diederichsen A, Rasmussen JM. Watermelon stomach and scleroderma. Ugeskrift for læger. 2002: 2047

12 Lin Y-T, Chuang Y-S, Wang J-W, Wu P-H. High risk of gastrointestinal hemorrhage in patients with systemic sclerosis. Arthritis research \& therapy. 2019: 1

13 Polkowska-Pruszyńska B, Gerkowicz A, Szczepanik-Kułak P, Krasowska D. Small intestinal bacterial overgrowth in systemic sclerosis: a review of the literature. Archives of dermatological research. 2019: 1 14 Brandler JB, Sweetser S, Khoshbin K, Babameto M, Prokop L, Camilleri M. Colonic manifestations and complications are relatively under-reported in systemic sclerosis: a systematic review. Official journal of the American College of Gastroenterology| ACG. 2019: 1847

15 Shah J, Shahidullah A. Chronic intestinal pseudo-obstruction in systemic sclerosis: an uncommon presentation. Case reports in gastroenterology. 2018: 373

16 Sattar B, Chokshi RV. Colonic and anorectal manifestations of systemic sclerosis. Current gastroenterology reports. 2019: 1

17 Govoni M, Muccinelli M, Panicali P, La Corte R, Scutellari PN, Orzincolo C, Pazzi P, Trotta F. Colon involvement in systemic sclerosis: clinical-radiological correlations. Clinical rheumatology. 1996: 271 18 Leighton JA, Valdovinos MA, Pemberton JH, Rath DM, Camilleri M. Anorectal dysfunction and rectal prolapse in progressive systemic sclerosis. Diseases of the colon \& rectum. 1993: 182

19 Thoua NM, Schizas A, Forbes A, Denton CP, Emmanuel AV. Internal anal sphincter atrophy in patients with systemic sclerosis. Rheumatology. 2011: 1596 
20 Assassi S, Fritzler MJ, Arnett FC, Norman GL, Shah KR, Gourh P, Manek N, Perry M, Ganesh D, Rahbar $\mathrm{MH}$. Primary biliary cirrhosis (PBC), PBC autoantibodies, and hepatic parameter abnormalities in a large population of systemic sclerosis patients. The Journal of rheumatology. 2009: 2250 